\title{
Estimating the number needed to vaccinate to prevent diseases and death related to human papillomavirus infection
}

\author{
Marc Brisson PhD, Nicolas Van de Velde MSc, Philippe De Wals MD PhD, Marie-Claude Boily PhD \\ $\infty \quad$ See related articles pages $456,462,469,480$ and 484
}

\section{ABSTRACT}

Background: A vaccine against human papillomavirus (HPV) types $6,11,16$ and 18 is now licensed for use in Canada and many other countries. We sought to estimate the number needed to vaccinate to prevent HPV-related diseases and death.

Methods: A cohort model of the natural history of HPV infection was developed. Model simulations were based on 209 different parameter sets that reproduced Canadian HPV type-specific data for infection, cervical intraepithelial neoplasia, cervical cancer and genital warts. The number needed to vaccinate was calculated as the number of women who would need to be vaccinated to prevent an HPV-related event during their lifetime.

Results: Among 12-year-old girls, we estimated that the number needed to vaccinate to prevent an episode of genital warts would be 8 ( $80 \%$ credibility interval $\left.[\mathrm{Crl}]^{-15}\right)$ and a case of cervical cancer 324 (80\% Crl 195-757). These estimates were based on the assumption that the vaccine procures lifelong protection and that its efficacy is $95 \%$. If vaccine protection is assumed to wane at $3 \%$ per year, the predicted number needed to vaccinate would increase to $14(80 \% \mathrm{Crl} 6-18)$ and 9080 ( $80 \% \mathrm{Crl} 1040-$ does not prevent), respectively. The latter number would be greatly reduced with the addition of a booster dose, to 480 ( $80 \% \mathrm{Crl} 254-1572)$.

Interpretation: Our model predictions suggest that vaccination with the currently available HPV vaccine may significantly reduce the incidence of genital warts, cervical intraepithelial neoplasia and cervical cancer. However, the benefits (particularly in terms of cervical cancer reduction) are highly dependent on the duration of vaccine protection, on which evidence is currently limited.

Une version française de ce résumé est disponible à l'adresse www.cmaj.ca/cgi/content/full/177/5/464/DCI

CMAJ 2007;177(5):464-8

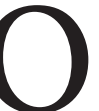
ver 40 human papillomavirus (HPV) genotypes are known to infect the anogenital tract. ${ }^{1}$ The International Agency for Research on Cancer has classified these types as being of either high oncogenic risk or low oncogenic risk according to their association with cervical cancer. ${ }^{2}$ About $70 \%$ of all cases of cervical cancer can be at- tributed to HPV types I6 and I8, ${ }^{2-4}$ which are of high oncogenic risk, and about $90 \%$ of all cases of genital warts are associated with HPV types 6 and $\mathrm{II},{ }^{5,6}$ which are of low oncogenic risk.

Two HPV prophylactic vaccines are currently in clinical trials: one targets HPV types 6, II, I6 and I8, and the other targets types 16 and I8. Recent large, randomized, placebo-controlled trials have shown that the one targeting types $6, \mathrm{II}, \mathrm{I} 6$ and $\mathrm{I} 8$ significantly reduced the incidence of type-specific anogenital and cervical lesions among women not previously infected with these virus strains. ${ }^{7,8}$ Given its efficacy and safety, this vaccine is now licensed for use in Canada and many other countries. ${ }^{9,10}$

"Number needed to treat" has been used to describe the population outcome of chronic disease treatments. By analogy, "number needed to vaccinate" has been used for vaccine preventable diseases. ${ }^{11}$ The number needed to vaccinate can be a helpful measure to illustrate the potential benefit of HPV vaccination, since it combines both the effect of vaccine efficacy and the age-specific background incidence of disease.

Because many of the benefits of prophylactic HPV vaccines will occur in the medium to long term, mathematical models are needed to project the impact of vaccination beyond the time horizon of clinical trials. The development of models are based on assumptions, which inevitably cause a level of uncertainty. In the case of HPV vaccination, it is particularly important to quantify the uncertainty surrounding model predictions, because the natural history of HPV is complex (it encompasses numerous stages of disease that depend on HPV type, screening and treatment) and the scientific literature on age- and type-specific natural history and transmission is limited. ${ }^{12}$ Furthermore, important questions remain regarding HPV prophylactic vaccines, such as the duration of vaccine protection and the overall efficacy of the vaccines against cervical cancer (since surrogate outcomes were used for cervical cancer in clinical trials). ${ }^{13}$ We sought to estimate the number needed to vaccinate to prevent HPV-related diseases and death and to quantify uncertainty around model predictions.

From the Département de médecine sociale et préventive (all authors), Université Laval; Unité de recherche en santé des populations (Brisson, Van de Velde), Centre hospitalier affilié universitaire de Québec, Québec, Que.; Unité de recherche en santé publique et santé environnementale (Van de Velde, De Wals), Centre hospitalier universitaire de Québec, Québec, Que.; and the Department of Infectious Disease Epidemiology (Boily), Imperial College, London, UK 


\section{Methods}

\section{Model structure}

We developed a model that follows a cohort of Io-year-old girls through different cervical infection and disease states (susceptible, infected, immune, genital warts, grade I cervical intraepithelial neoplasia, grade 2 or 3 cervical intraepithelial neoplasia, cervical cancer) for 4 classes of HPV genotypes (type I6, type I8, other types of high oncogenic risk, and types of low oncogenic risk). The structure we used is identical to a previously published model ${ }^{12}$ except that we included the natural history of genital warts. The model is static and therefore does not take into account herd-immunity effects following vaccination.

Model assumptions were as follows: there is no crossprotection between HPV types; transition rates between disease states are type- and age-specific; co-infections with 2 HPV types can occur; women infected with 2 HPV types follow the progression and regression rates of the most aggressive type; and lifelong immunity can develop following infection with HPV type 16 or 18 but not with other HPV types of high oncogenic risk or with types of low oncogenic risk. The model also accounts for screening and treatment outcomes. Women have an age-specific probability of being screened, and screening tests have a lesion-specific sensitivity of detecting cervical disease.

\section{Model parameters}

Demographic, screening and treatment parameters were estimated from available Canadian data; otherwise US data were used. ${ }^{12}$ All estimates of natural history parameters were specific to the 4 classes of HPV (type I6, type I8, other types of high oncogenic risk, and types of low oncogenic risk) and to age. An extensive fitting procedure was performed to identify different sets of natural history parameters. Model fit was performed in 4 steps (these have been described in detail previously ${ }^{12}$ ). First, we conducted a literature search of prospective HPV studies published between 1995 and 2005. Each type-specific natural history parameter was assigned a prior uniform distribution between the minimum and maximum estimates found in the literature review (for prior distributions of parameter values and references, see Appendix I, available online at www.cmaj.ca/cgi/content/full/I77 15/464/DC2). Second, 200 ooo parameter sets were drawn from the uniform prior parameter distributions. Third, epidemiologic data specific to North America were identified, through a review of articles and reports, for model fit. ${ }^{12-15}$ Whenever possible, data specific to Canada were used. Finally, parameter sets from step 2 were judged to produce "acceptable" fit if the associated model predictions fell simultaneously within pre-specified targets defined using the epidemiologic data from step 3. Of the 200000 randomly sampled combinations of parameters, 209 sets of natural history parameters met our predefined goodness-of-fit criteria for prevalence and annual incidence of HPV, genital warts, cervical intraepithelial neoplasia and cervical cancer. (The fit criteria are described by Van de Velde and associates, ${ }^{12}$ and the parameter values appear in Appendix I, available online at www .cmaj.ca/cgi/content/full/177/5/464/DC2.) The 209 identified parameter sets can loosely be considered as different models that allow thorough investigation of the impact of natural history assumptions and parameter uncertainty on model predictions.

\section{Model simulations}

Model simulations were based on the 209 posterior parameter sets identified during model fitting. The number needed to vaccinate is the number of women (within a specific age cohort) who would need to be vaccinated to prevent a single HPV-related event during their lifetime. The number needed to vaccinate $(\mathrm{NNV})$ is calculated as follows: $\mathrm{NNV}=\mathrm{N} \div \mathrm{P}^{1}$, where $\mathrm{N}$ is the size of the vaccinated cohort, and $\mathrm{P}$ is the predicted number of HPV-related events prevented in the vaccinated cohort over its lifetime. In the case of the number needed to vaccinate to gain a life-year, we divided the size of the vaccinated cohort by the total number of life-years gained in the cohort by preventing deaths from cervical cancer. Results are expressed as the mean and $80 \%$ credibility interval (Ioth and goth percentiles) of the 209 simulations. (Credibility intervals are the Bayesian analog to confidence intervals.)

\section{Vaccine characteristics}

For the base case, we made the following assumptions about the vaccine: initial vaccine efficacy is $95 \%$, and vaccine duration is lifelong. These assumptions are based on results from clinical trials that showed that prophylactic HPV vaccines were highly effective for at least 5 years, that there is no evidence of waning efficacy and that the vaccine induces robust immune memory, which is the hallmark of long-term protection. ${ }^{16,17}$ Similar to other HPV vaccine modelling studies, ${ }^{18-21}$ we assumed that the duration of vaccine protection in the base case is lifelong. However, given the substantial uncertainty around this parameter, we varied the mean duration of protection between 30 years (assuming vaccine protection wanes at $3 \%$ per year) and life (previous antibody decay models, based on trial data for HPV type I6, have predicted that the median duration of detectable vaccineinduced serum anti-HPV-I6 levels following vaccination range from 32 years to life ${ }^{22}$ ). We also present a worst case in which the mean duration of vaccine protection is Io years. Duration is varied by assuming a constant waning of vaccine protection. In the sensitivity analysis, we varied vaccine efficacy between $70 \%$ and $100 \%$ to represent the $95 \%$ confidence intervals of the per protocol vaccine efficacy against HPV types 6 , II, I6 and I8 $(70 \%-97 \%)$ and types 16 and $18(8 \mathrm{I} \%-100 \%) .{ }^{23,24}$

Because we were modelling prophylactic HPV vaccination, we did not include any therapeutic benefits to women already infected with HPV types targeted by the vaccine. Furthermore, we assumed that the natural history of disease would be unaltered following vaccine failure or loss of vaccine-induced immunity.

\section{Vaccine strategies}

For the base-case vaccine strategy, we assumed that the age at vaccination is 12 years. Sensitivity analysis was performed to evaluate the impact of age at vaccination and receipt of a booster dose (assumed to be at 22 years of age if duration of protection is less than lifelong).

\section{Results}

The numbers needed to vaccinate to prevent outcomes related to HPV infection in a cohort of 12 -year-old girls are shown in Table $\mathrm{I}$. The number needed to vaccinate to prevent an epi- 
sode of genital warts is low because I in Io women are estimated to get genital warts in their lifetime,${ }^{14}$ many individuals have more than one episode of genital warts, ${ }^{14}$ and about $90 \%$ of cases are associated with HPV types 6 and II, which are the HPV types of low oncogenic risk included in the vaccine currently licensed for use in Canada. ${ }^{5,6}$ The number needed to vaccinate to prevent a life-year lost is also low because death from cervical cancer can occur in young women, and therefore preventing a death provides important gains in life-years.

If vaccine protection is assumed to wane at $3 \%$ per year, the number needed to vaccinate increases dramatically (particularly for cervical cancer) and results become highly variable (Table I, Figure I, and online Appendix 2, available at www .cmaj.ca/cgi/content/full/177/5/464/DC2). In a worst case scenario (age at vaccination 12 years, vaccine efficacy $70 \%$, waning vaccine protection $3 \%$ per year), the number needed to vaccinate to prevent an episode of genital warts is estimated to be 18 and the lifetime reduction of cervical cancer is $\% \%$ (results not shown). The impact of duration of vaccine protection is due to shifts in the age at infection. A vaccine with limited duration is predicted to prevent natural infection at the beginning of sexual activity and move the pool of susceptible people toward higher ages. If the force of HPV infection remains high among older women or progression rates toward cervical cancer are greater among older women, then waning immunity can have an important effect on the number needed to vaccinate. The number needed to vaccinate and the level of uncertainty around the model predictions are greatly reduced with the addition of a booster dose (Table I, Figure I, and online Appendix 2, available at www.cmaj.ca/cgi/content/full/177/5/464/DC2).

The predicted numbers needed to vaccinate are relatively insensitive to vaccine efficacy above $70 \%$ and vaccination at age 15 or 20 years (Figure I). However, the predicted numbers and related uncertainty increase when "older" women are vaccinated. Predictions for older women depend on the proportion of HPV- related disease that is attributable to infection at higher ages. The increased uncertainty of predictions among women vaccinated at 25 years of age reflects our limited understanding of the epidemiology of HPV infection and disease in "older" women.

\section{Interpretation}

We used a mathematical model to estimate the numbers needed to vaccinate to prevent HPV-related diseases and death and to quantify the uncertainty around the predictions. When vaccine protection is assumed to be lifelong, the predicted numbers needed to vaccinate are low. This prediction reflects the high efficacy of prophylactic HPV vaccination reported in the clinical trials and the fact that the annual incidence of HPV-related diseases remains high in Canada despite current screening programs. However, if the mean duration of protection conferred by HPV vaccination is less than 30 years, the efficacy of the vaccine at preventing cervical cancer is predicted to be limited, unless booster doses are given.

Comparisons of numbers needed to vaccinate between vaccines for different infections or diseases should be performed with great care. Results must be compared using the same timeframe of analysis and with similar outputs (e.g., a case of genital warts is not equal to a case of cervical cancer). For example, on the basis of the results of the FUTURE II randomized control trial, ${ }^{8}$ Sawaya and Smith-McCune ${ }^{13}$ estimated that 129 women would need to be vaccinated to prevent a case of grade 2 or 3 cervical intraepithelial neoplasia or adenocarcinoma in situ. In contrast, we estimated that between 8 and 43 girls aged I2 would need to be vaccinated to prevent a case of grade 2 or 3 cervical intraepithelial neoplasia (the number depending on the mean duration of vaccine protection). The results differ because our estimates were assessed over the duration of vaccine efficacy, whereas those from Sawaya and Smith-McCune ${ }^{13}$ were over the first 3 years following HPV vaccination.

Table 1: Estimated numbers needed to vaccinate with quadrivalent human papillomavirus (HPV) vaccine* to prevent HPV-related diseases and death in a cohort of 12-year-old girls, by duration of vaccine protection and efficacy of vaccine

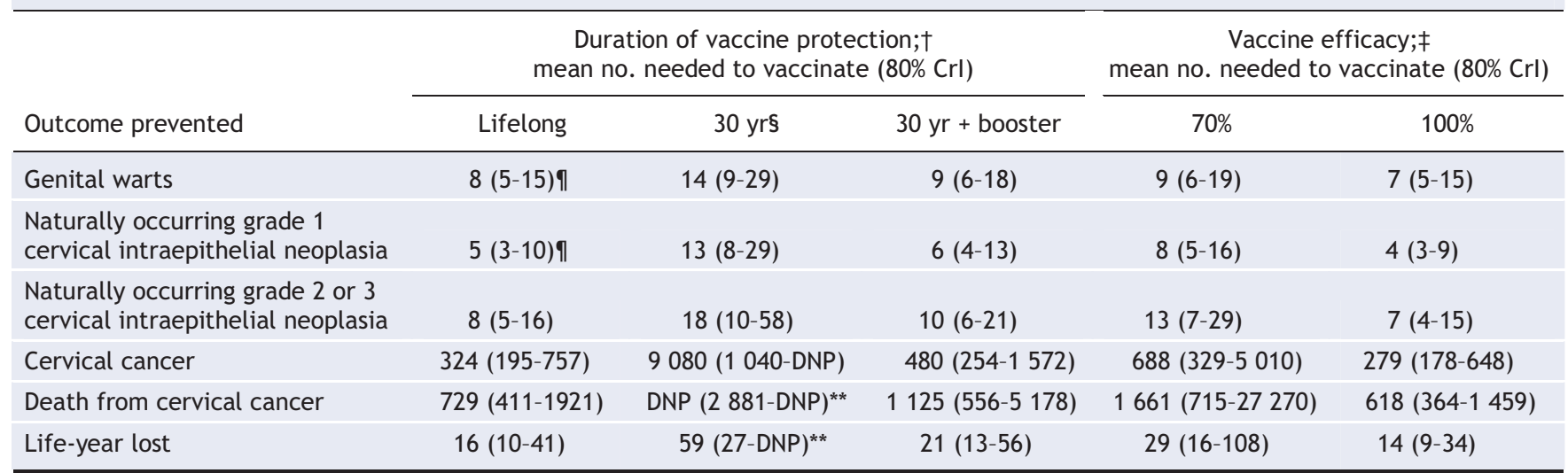

Note: $\mathrm{Crl}=$ credibility interval, DNP = does not prevent.

*Vaccine used in model targets HPV genotypes 6, 11, 16 and 18.

†Assuming vaccine efficacy is $95 \%$.

$\ddagger$ Assuming duration of vaccine protection is lifelong.

$\S$ The mean duration of vaccine protection of 30 years corresponds to a vaccine waning rate of $3.3 \%$ per year.

IIf a vaccine that targets HPV types 16 and 18 is used, the number needed to vaccinate to prevent a case of naturally occurring grade 1 cervical intraepithelial neoplasia

is predicted to be 6 and genital warts would not be prevented. All other estimates would be identical to those with the vaccine used in the model.

** The model predicts that HPV vaccine does not prevent death from cervical cancer but, rather, delays the onset of cancer and thus produces life-year gains. 
To put the results of our analysis into perspective, we compared the HPV vaccine with 3 other vaccines (varicella, meningococcal and influenza vaccines) in terms of the number needed to treat to prevent I death. For varicella vaccination, we estimated that 34000 people would need to be vaccinated to prevent I death (using mortality rates reported by Brisson and Edmund ${ }^{25}$ and assuming 100\% efficacy against the varicella-zoster virus and no waning in protection). To prevent $\mathrm{I}$ meningococcalrelated death, we estimated that about 2I 000 people would need to be vaccinated (using mortality data reported by De Wals and associates ${ }^{26}$ and assuming $100 \%$ efficacy against types $\mathrm{A}, \mathrm{C}$, $\mathrm{Y}$ and Wi35). Finally, we estimated that 5000 people would need to be vaccinated to prevent I death from influenza (assuming the vaccine is given to people aged 65 or more). ${ }^{11}$ These results are higher than our estimates for the prevention of a death from cervical cancer with HPV vaccination when we assume that the duration of protection is lifelong or that a booster dose is given to women with waning vaccine-induced immunity. However, if vaccine protection is assumed to wane at $3 \%$ per year (mean protection 30 years, efficacy $95 \%$ ), the estimated number needed to vaccinate with HPV vaccine is unlikely to be lower than those for varicella, meningococcal and influenza vaccines. Only $23 \%$ and $45 \%$ of the model simulations (using the 209 posterior parameter sets) predicted that the number of I2-year-old girls who would need to be vaccinated to prevent I HPV-related death would be less than 5000 (number needed to prevent I influenza-related death) and 34000 (number needed to prevent I varicella-related death), respectively.

The long-term efficacy for a new vaccine is usually unknown owing to the relatively short duration of clinical trials. Current studies suggest that HPV vaccines used for prophylaxis are highly effective for at least 5 years and that there is no evidence of waning efficacy during that period. ${ }^{16}$ However, the long-term duration of vaccine-induced immunity remains unclear. Given the importance of the duration of protection on the effectiveness of HPV vaccination, post-vaccination surveillance of waning efficacy is essential. If the protection of the HPV vaccine does wane substantially, booster shots will likely be required to maintain and lengthen vaccine-induced immunity. Our model predicts that a booster dose would significantly reduce the number needed to vaccinate and would increase the effectiveness of HPV vaccination if waning occurs. However, uptake (coverage) of the booster dose would probably be lowest among women who would need it the most, because they will likely be the same women who are not regularly screened for cervical cancer.
The main limitation of our study is that the model does not account for herd-immunity effects. Previous modelling studies have predicted that, if coverage rates are high, vaccination will produce positive herd-immunity effects. ${ }^{18,19,27}$ Hence, if high coverage rates are attained (which would be expected in Canada with routine immunization), the population-based number needed to vaccinate will likely be lower than the estimates in our study. On the other hand, if coverage rates are low or if our esti-

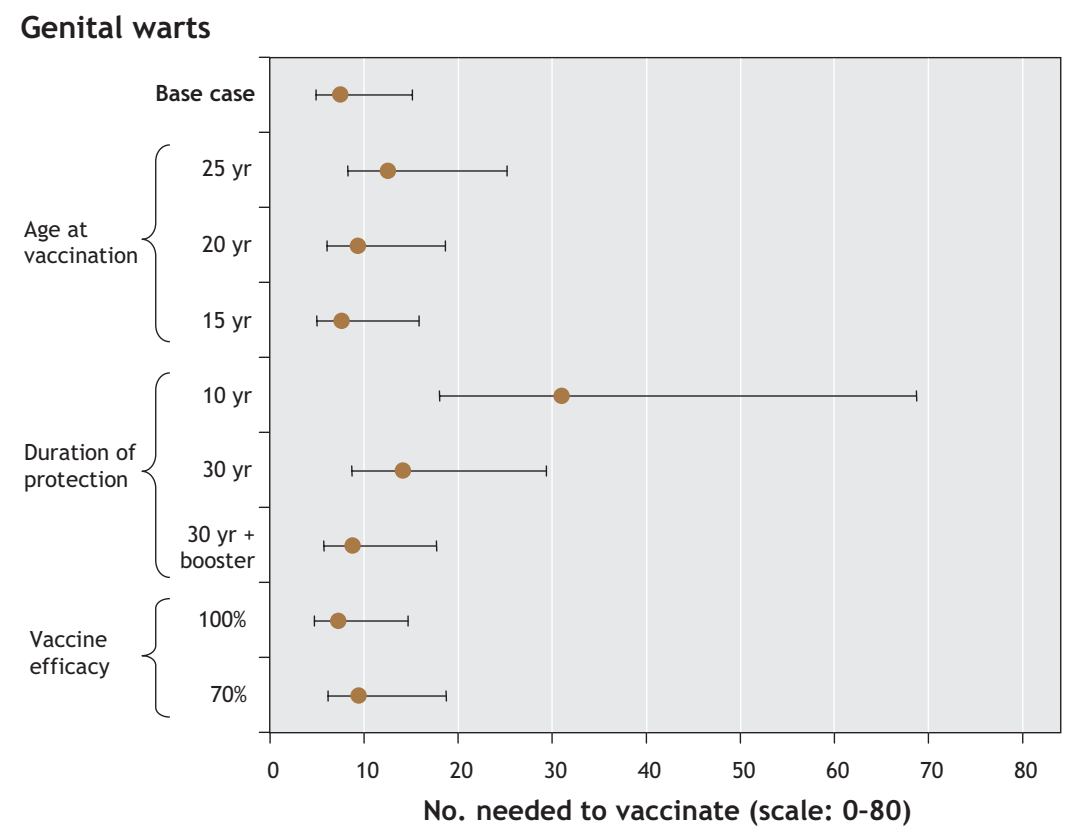

Cervical cancer

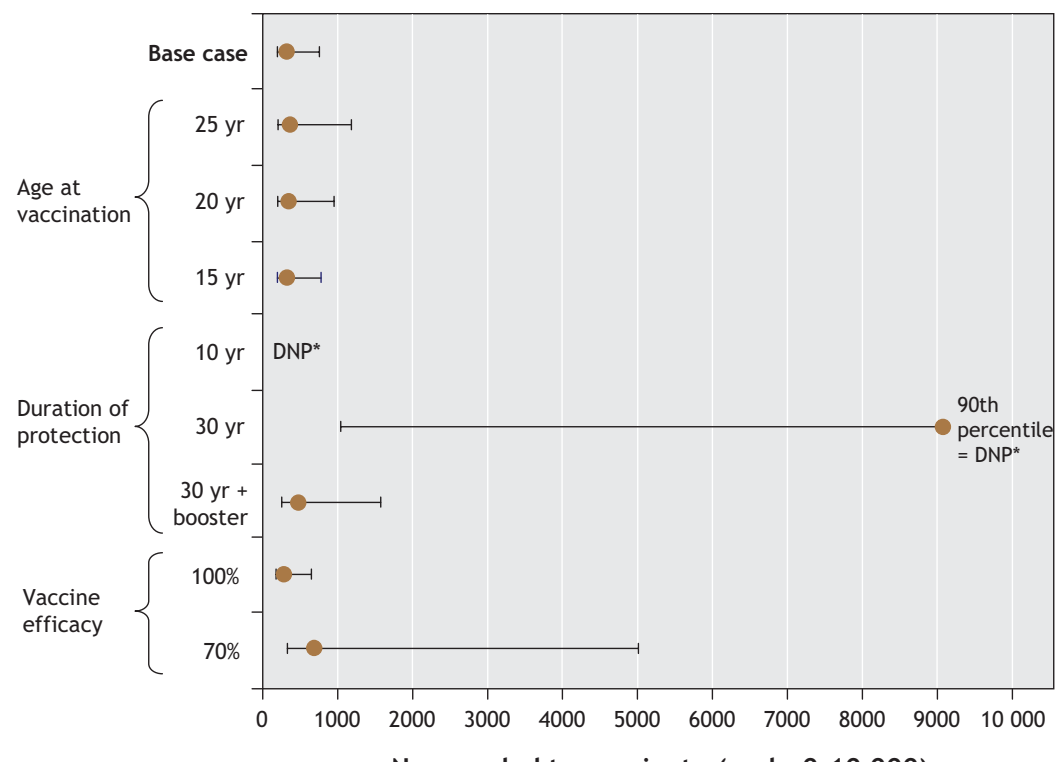

No. needed to vaccinate (scale:0-10 000)

Figure 1: Sensitivity analysis of 209 different parameter sets that reproduced Canadian data specific to human papillomavirus (HPV) type for infection, cervical intraepithelial neoplasia, cervical cancer and genital warts. These graphs plot the numbers needed to vaccinate to prevent an episode of genital warts and a case of cervical cancer. For the base case, it is assumed that the vaccine efficacy is $95 \%$, the duration of protection is lifelong and the age at vaccination is 12 years. ${ }^{*} \mathrm{DNP}=$ does not prevent outcome. 
mates are applied only to those who are vaccinated, accounting for herd-immunity effects will have little or no effect on predictions. Our estimates should be generalized only to settings or countries with similar HPV epidemiologic data, because the estimates are dependent on the age-specific background rates of disease. Another limitation is that the number needed to vaccinate was calculated from mean population rates of HPV infection and disease. Hence, it may not represent subgroups within Canada that have different HPV epidemiology and screening rates (e.g., high-risk groups). Finally, we focused on the number needed to vaccinate to prevent an episode of genital warts, a case of cervical intraepithelial neoplasia and a case of cervical cancer because they are currently the most important HPV-related diseases in terms of death and use of health care resources. However, for a complete picture of the potential benefits of HPV vaccination, one should include the predicted number needed to vaccinate to prevent other anogenital cancers, head and neck cancers, and recurrent respiratory papillomatosis.

The main strength of our study is that we performed an extensive fitting procedure and identified multiple parameter sets that reproduced available epidemiologic data, which allowed us to illustrate the uncertainty around model predictions. The wide credibility intervals when efficacy is assumed to wane or vaccination is given to "older" women reflect the uncertainty in the natural history of HPV in older adults and suggest that more research is needed in this area. Although challenging owing to the lack of long-term data, more studies should be focused on quantifying the rate of waning protection following vaccination.

In summary, our model illustrates the potential benefit of HPV vaccination by combining both the effect of vaccine efficacy and the background incidence of HPV-related disease. Our model predictions suggest that prophylactic use of the vaccine against HPV types 6, II, I6 and I8 has the potential to significantly reduce the incidence of genital warts, cervical intraepithelial neoplasia and cervical cancer. However, the benefits (particularly in terms of cervical cancer reduction) are highly dependent on the duration of vaccine protection, on which evidence is currently limited. Recommendations regarding HPV vaccination should take into account the uncertainty regarding long-term vaccine efficacy. If mass HPV vaccination is implemented, cervical cancer screening must continue among vaccinated women, and careful long-term post-vaccination surveillance of vaccine fficacy will be essential.

\section{This article has been peer reviewed.}

Competing interests: None declared for Nicolas Van de Velde. Marc Brisson was an employee of Merck Frosst Canada Ltd. during the analysis and writing of the first draft of the paper. He has consulted for Merck Frosst and has received reimbursement for travel expenses from GlaxoSmithKline. Philippe De Wals has received research grants, reimbursement for travel expenses and honoraria for conferences from vaccine manufacturers, including Aventis Pasteur, GlaxoSmithKline, Shire, Chiron, Baxter, Merck Frosst, and Wyeth-Ayerst. Marie-Claude Boily has received an unrestricted grant from Merck Frosst Canada Ltd. for research on the impact of human papillomavirus vaccination.

Contributors: Marc Brisson was responsible for the concept and design of the study. He along with Nicolas Van De Velde and Marie-Claude Boily analyzed and interpreted the data and drafted the manuscript. Marc Brisson, Philippe De Wals and Marie-Claude Boily critically revised the manuscript for important intellectual content. All of the authors approved the version to be published.

\section{REFERENCES}

I. De Villiers EM, Fauquet C, Broker TR, et al. Classification of papillomaviruses. Virology 2004;324:17-27.

2. Munoz N, Bosch FX, de Sanjose S, et al. Epidemiologic classification of human papillomavirus types associated with cervical cancer. NEngl JMed 2003;348:518-27.

3. Burd EM. Human papillomavirus and cervical cancer. Clin Microbiol Rev 2003; I6: I-I7.

4. Clifford GM, Smith JS, Aguado T, et al. Comparison of HPV type distribution in high-grade cervical lesions and cervical cancer: a meta-analysis. Br J Cancer 2003; 89:IOI-5.

5. Greer CE, Wheeler CM, Ladner MB, et al. Human papillomavirus (HPV) type distribution and serological response to HPV type 6 virus-like particles in patients with genital warts. J Clin Microbiol I995;33:2058-63.

6. Von Krogh G, Lacey CJ, Gross G, et al. European course on HPV associated pathology: guidelines for primary care physicians for the diagnosis and management of anogenital warts. Sex Transm Infect 2000;76:162-8.

7. Garland SM, Hernandez-Avila M, Wheeler CM, et al. Quadrivalent vaccine against human papillomavirus to prevent anogenital diseases. $N$ Engl J Med 2007;356: I928-43.

8. FUTURE II Study Group. Quadrivalent vaccine against human papillomavirus to prevent high-grade cervical lesions. N Engl J Med 2007;356:1915-27.

9. Center for Biologics Evaluation and Research, US Food and Drug Administration. Product approval information: human papillomavirus (types 6, II, I6, I8) recombinant vaccine (Gardasil). STN: I25126/0. Available: www.fda.gov/cber/products /gardasil.htm (accessed 2007 Jul 20).

Io. Health Canada. Drugs and Health Products. Notice of compliance (NOC): Gardasil; recombinant human papillomavirus vaccine (types 6, II, I6, I8). DIN: 02283190. Available: www.hc-sc.gc.ca/dhp-mps/alt_formats/hpfb-dgpsa/txt/prodpharma /bio2006et.txt (accessed 2007 July 4).

II. Kelly H, Attia J, Andrews R, et al. The number needed to vaccinate (NNV) and population extensions of the NNV: comparison of influenza and pneumococcal vaccine programmes for people aged 65 years and over. Vaccine 2004;22:2192-8.

I2. Van de Velde N, Brisson M, Boily MC. Modeling human papillomavirus vaccine effectiveness: quantifying the impact of parameter uncertainty. Am J Epidemiol 2007; I65:762-75.

I3. Sawaya GF, Smith-McCune K. HPV vaccination - more answers, more questions. NEngl J Med 2007;356:I99I-3.

I4. Insinga RP, Dasbach EJ, Myers ER. The health and economic burden of genital warts in a set of private health plans in the United States. Clin Infect Dis 2003;36: I397-403.

I5. Winer RL, Kiviat NB, Hughes JP, et al. Development and duration of human papillomavirus lesions, after initial infection. JInfect Dis 2005;I9I:73I-8.

I6. Villa LL, Costa RL, Petta CA, et al. High sustained efficacy of a prophylactic quadrivalent human papillomavirus types $6 / \mathrm{II} / \mathrm{I} 6 / \mathrm{I} 8 \mathrm{LI}$ virus-like particle vaccine through 5 years of follow-up. BrJ Cancer 2006;95:1459-66.

I7. Olsson SE, Villa LL, Costa RL, et al. Induction of immune memory following administration of a prophylactic quadrivalent human papillomavirus (HPV) types 6/II/I6/I8 Li virus-like particle (VLP) vaccine. Vaccine 2007;25:493I-9.

I8. Elbasha EH, Dasbach EJ, Insinga RP. Model for assessing human papillomavirus vaccination strategies. Emerg Infect Dis 2007;13:28-4I.

19. French KM, Barnabas RV, Lehtinen M, et al. Strategies for the introduction of human papillomavirus vaccination: modelling the optimum age- and sex-specific pattern of vaccination in Finland. BrJ Cancer 2007;96:514-8.

20. Goldie SJ, Kohli M, Grima D, et al. Projected clinical benefits and cost-effectiveness of a human papillomavirus I6/I8 vaccine. J Natl Cancer Inst 2004;96:604-I5.

2I. Kohli M, Ferko N, Martin A, et al. Estimating the long-term impact of a prophylactic human papillomavirus I6/I8 vaccine on the burden of cervical cancer in the UK. BrJ Cancer 2007;96:143-50.

22. Fraser $\mathrm{C}$, Tomassini JE, Xi L, et al. Modeling the long-term antibody response of human papillomavirus (HPV) virus-like particle (VLP) type 16 prophylactic vaccine. Vaccine 2007;25:4324-33.

23. Villa LL, Costa RL, Petta CA, et al. Prophylactic quadrivalent human papillomavirus (types 6, II, I6, and I8) Li virus-like particle vaccine in young women: a randomised double-blind placebo-controlled multicentre phase II efficacy trial. Lancet Oncol 2005;6:27I-8.

24. Harper DM, Franco EL, Wheeler CM, et al. Sustained efficacy up to 4.5 years of a bivalent LI virus-like particle vaccine against human papillomavirus types $\mathrm{I} 6$ and I8: follow-up from a randomised control trial. Lancet 2006;367:1247-55.

25. Brisson M, Edmunds WJ. The cost-effectiveness of varicella vaccination in Canada. Vaccine 2002;20:1113-25.

26. De Wals P, Coudeville L, Trottier P, et al. Vaccinating adolescents against meningococcal disease in Canada: a cost-effectiveness analysis. Vaccine 2007;25:5433-40.

27. Taira AV, Neukermans CP, Sanders GD. Evaluating human papillomavirus vaccination programs. Emerg Infect Dis 2004;10:1915-23

Correspondence to: Dr. Marc Brisson, Unité de recherche en santé des populations, Centre hospitalier affilié universitaire de Québec, Hôpital Saint-Sacrement, I050, chemin Sainte-Foy, Québec QC GIS 4L8; marc.brisson@uresp.ulaval.ca 